TRANSACTIONS OF THE

AMERICAN MATHEMATICAL SOCIETY

Volume 360, Number 7, July 2008, Pages 3557-3578

S 0002-9947(08)04327-4

Article electronically published on January 9, 2008

\title{
HÖLDER REGULARITY OF THE SLE TRACE
}

\author{
JOAN R. LIND
}

\begin{abstract}
S. Rohde and O. Schramm have recently shown that the SLE trace is Hölder continuous (2005). However, their results are not optimal for all values of $\kappa$ and only yield a Hölder exponent near $\frac{1}{2}$ for $\kappa$ near 0 . In this paper, we give improved lower bounds on the optimal Hölder exponent for two natural parametrizations of the SLE trace. Our estimates give a Hölder exponent near 1 for $\kappa$ near 0, as expected. The work of I. Binder and B. Duplantier (2002) suggests that our results are optimal for the two parametrizations considered.
\end{abstract}

\section{INTRODUCTION}

In studying the question of whether the loop-erased random walk has a conformally invariant scaling limit, O. Schramm [10] was led to introduce SLE, which is a one parameter family of continuous random processes. Originally named "Stochastic Loewner Evolution" by O. Schramm, SLE is now referred to as "SchrammLoewner Evolution." One can think of $\mathrm{SLE}_{\kappa}$ as a random curve, often called the "trace", in the upper halfplane, which was generated via Loewner's differential equation with a random driving term. We will describe this in more detail in the following section.

From the work of S. Rohde and O. Schramm in [9], we know that the SLE trace is Hölder continuous for $\kappa \neq 8$. However the exponents they obtain are clearly not optimal for $\kappa$ near 0 . (When $\kappa=0$, the trace is a straight line which meets the real line perpendicularly, as pictured in Figure 2, It is this right angle which causes problems for the natural parametrizations of the trace.) This gives rise to the question as to what the optimal Hölder exponent is. There is an upper bound for this quantity from the recent work of V. Beffara [1], who has shown that the Hausdorff dimension of the trace is $1+\frac{\kappa}{8}$ for $\kappa<8, \kappa \neq 4$. Thus, the Hölder exponent for the trace cannot be more than the reciprocal of this, which is $\frac{8}{8+\kappa}$ for $\kappa<8$. For $\kappa \geq 8$, the trace is a space-filling curve a.s. 9], giving an upper bound of $\frac{1}{2}$ for the Hölder exponent.

In this paper, we will see that the estimates of [9] can be improved on, as long as we stay away from the base of the curve. To accomplish this, we work in a slightly different setting, where we no longer have the problem of the angle at the base of the curve. Applying the techniques of [9] in this setting yield better Hölder exponents for the revised trace, which carry back over to our original trace as long as we stay away from its base. Following this strategy gives the following two results. The latter is a corollary of Theorem 3 which gives the Hölder continuity of the conformal map $f_{t}$ away from the driving term.

Received by the editors February 24, 2005 and, in revised form, April 22, 2006.

2000 Mathematics Subject Classification. Primary 60D05, 30C35, 60G17. 


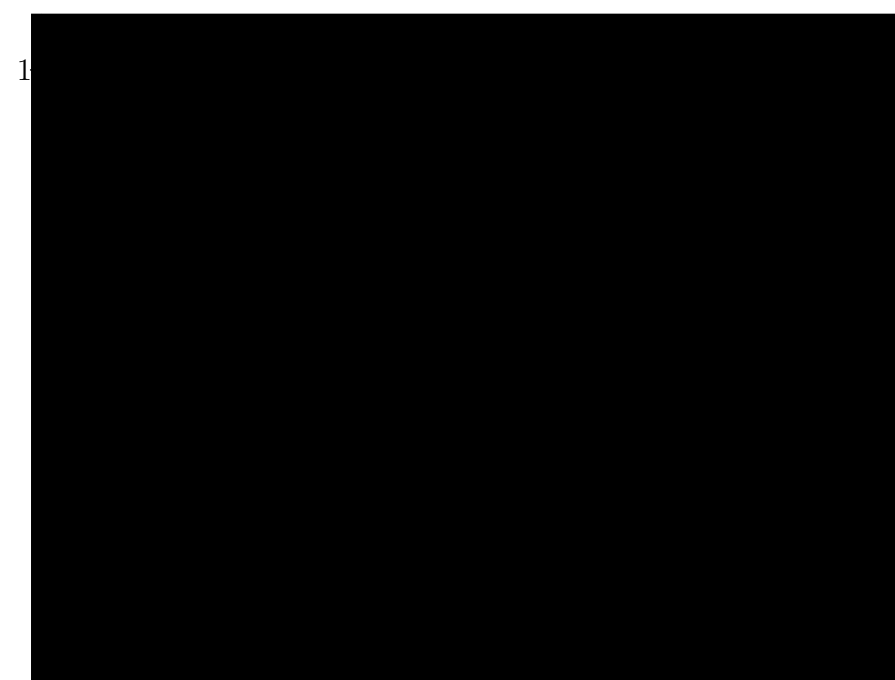

Figure 1. The functions $\sigma(\kappa)$ and $\eta(\kappa)$. Important features to note about $\sigma$ are that $\sigma(0)=1, \sigma(8)=0$, and $\lim _{\kappa \rightarrow \infty} \sigma(\kappa)=1 / 2$. For $\eta$, we have that $\eta(0)=1, \eta(4)=0$, and $\lim _{\kappa \rightarrow \infty} \eta(\kappa)=1$.

Theorem 1. Consider any subcurve of the $S L E_{\kappa}$ trace in $\mathbb{H}$ which does not intersect a disc of fixed positive radius centered at 0 . Then there is a natural parametrization of this curve which is Hölder continuous with exponent $\sigma-\epsilon$, where $\epsilon>0$ and

$$
\sigma(\kappa)=1-\frac{\kappa}{2 \kappa+24-8 \sqrt{\kappa+8}} .
$$

Theorem 2. Let $\kappa<4$. Consider any bounded subcurve of the $S L E_{\kappa}$ trace in $\mathbb{H}$ which does not intersect a disc of fixed positive radius centered at 0 . Then there is a natural parametrization of this curve which is Hölder continuous with exponent $\eta-\epsilon$, where $\epsilon>0$ and

$$
\eta(\kappa)=2-\frac{(\kappa+4)^{2}}{\kappa^{2}+12 \kappa+16-2 \sqrt{2 \kappa(\kappa+2)(\kappa+8)}} .
$$

See Figure 1 for graphs of $\sigma(\kappa)$ and $\eta(\kappa)$.

The paper is organized as follows: In the first section, we introduce the Loewner differential equation in the upper halfplane and chordal SLE. This is followed by a discussion of two natural parametrizations of the trace and the previous results for the Hölder continuity of each. Then we have a section containing some needed lemmas. In the last two sections, we prove two regularity theorems which imply the theorems above.

We recently became aware of D Beliaev's thesis 2 which could be used to derive Theorem 2

\section{The Loewner EQuation in the haLfPlane AND CHORDAL SLE}

Let $\gamma(t)$ be a simple continuous curve in $\mathbb{H} \cup\{0\}$ with $\gamma(0)=0$ and $t \in[0, T]$. Then there is a unique conformal map $g_{t}: \mathbb{H} \backslash \gamma[0, t] \rightarrow \mathbb{H}$ with the following 
normalization, called the hydrodynamic normalization, near infinity:

$$
g_{t}(z)=z+\frac{c(t)}{z}+O\left(\frac{1}{z^{2}}\right) .
$$

It is an easy exercise to check that $c(t)$ is continuously increasing in $t$ and that $c(0)=0$. Therefore $\gamma$ can be reparametrized so that $c(t)=2 t$. Assuming this normalization, one can show that $g_{t}$ satisfies the following form of Loewner's differential equation: for all $t \in[0, T]$ and all $z \in \mathbb{H} \backslash \gamma[0, t]$,

$$
\begin{aligned}
\frac{\partial}{\partial t} g_{t}(z) & =\frac{2}{g_{t}(z)-\lambda(t)}, \\
g_{0}(z) & =z,
\end{aligned}
$$

where $\lambda$ is a continuous, real-valued function. Further, it can be shown that $g_{t}$ extends continuously to $\gamma(t)$ and $g_{t}(\gamma(t))$ equals $\lambda(t)$.

On the other hand, if we start with a continuous $\lambda:[0, T] \rightarrow \mathbb{R}$, we can consider the following initial value problem for each $z \in \mathbb{H}$ :

$$
\begin{aligned}
\frac{\partial}{\partial t} g(t, z) & =\frac{2}{g(t, z)-\lambda(t)}, \\
g(0, z) & =z .
\end{aligned}
$$

For each $z \in \mathbb{H}$ there is some time interval $[0, s)$ for which a solution $g(t, z)$ exists. Let $T_{z}=\sup \{s \in[0, T]: g(t, z)$ exists on $[0, s)\}$. Set $G_{t}=\left\{z \in \mathbb{H}: T_{z}>t\right\}$ and $g_{t}(z)=g(t, z)$. Then one can prove that the set $G_{t}$ is a simply connected subdomain of $\mathbb{H}$ and $g_{t}$ is the unique conformal map from $G_{t}$ onto $\mathbb{H}$ with the following normalization near infinity:

$$
g_{t}(z)=z+\frac{2 t}{z}+O\left(\frac{1}{z^{2}}\right) .
$$

The function $\lambda(t)$ is called the driving term, and the domains $G_{t}$ as well as the functions $g_{t}$ are said to be generated by $\lambda$.

The domains $G_{t}$ generated by a continuous driving term $\lambda$ are not necessarily slit-halfplanes, i.e. domains of the form $\mathbb{H} \backslash \gamma[0, t]$, for some simple continuous curve $\gamma$ in $\mathbb{H} \cup\{\gamma(0)\}$ with $\gamma(0) \in \mathbb{R}$. See [7] for a condition which guarantees the generation of slit-halfplanes. The necessary and sufficient condition for a decreasing family of domains $\left\{G_{t}\right\}$ to be generated by a continuous driving term can be found in Section 2.3 of [5].

The simplest example is when the driving term is constant. If we take $\lambda(t)=0$, then the functions generated by the Loewner equation are $g_{t}(z)=\sqrt{z^{2}+4 t}$, and these map from the upper halfplane minus a vertical slit of length $2 \sqrt{t}$ onto the upper halfplane. See Figure 2 .

There is another version of the Loewner equation in the halfplane. Let $\xi$ : $[0, T] \rightarrow \mathbb{R}$ be continuous and consider the following initial value problem, in which a negative sign has been introduced on the right hand side of (1):

$$
\begin{aligned}
\frac{\partial}{\partial t} f(t, z) & =\frac{-2}{f(t, z)-\xi(t)}, \\
f(0, z) & =z
\end{aligned}
$$

for $z \in \mathbb{H}$. In this case, for each $z \in \mathbb{H}$, the solution $f(t, z)$ exists for all $t \in[0, T]$. Setting $f_{t}(z)=f(t, z)$, we have that $f_{t}$ is defined on all of $\mathbb{H}$. As in the previous 


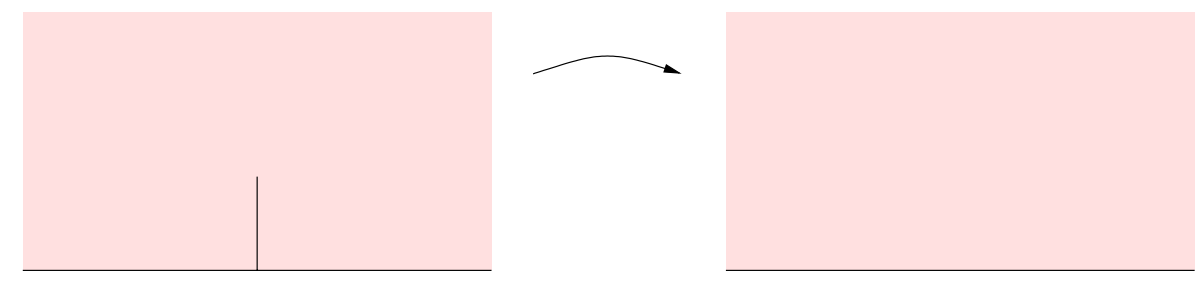

0

Figure 2. The function $g_{t}$ generated by the constant driving term $\lambda=0$ maps $\mathbb{H} \backslash[0,2 i \sqrt{t}]$ onto $\mathbb{H}$.

case, it can be shown that $f_{t}$ is a conformal map from $\mathbb{H}$ into $\mathbb{H}$, and near infinity it has the form

$$
f_{t}(z)=z+\frac{-2 t}{z}+O\left(\frac{1}{z^{2}}\right) .
$$

We think of the functions $f_{t}$ as being generated by "running time backward," and we will refer to equation (2) as the "backward Loewner equation."

These two forms of Loewner's differential equation in the halfplane are related. Given a continuous function $\lambda$ on $[0, T]$, set $\xi(t)=\lambda(T-t)$. Let $g_{t}$ be the functions generated by $\lambda$ from (11), and let $f_{t}$ be the functions generated by $\xi$ from (2). It is not true that $f_{t}(z)=g_{t}^{-1}(z)$ for all $t \in[0, T]$, but it is true that $f_{T}(z)=g_{T}^{-1}(z)$.

The halfplane version is often called the chordal Loewner equation because it generates compact sets, $K_{t}=\mathbb{H} \backslash G_{t}$, which grow from 0 toward infinity, that is, they grow from one boundary point toward another. The sets $K_{t}$ are often called "hulls." Another standard version of the Loewner equation is the disk version, often called the radial Loewner equation, which generates compact sets that grow from a point on the boundary toward the center. In this paper we restrict our attention to the chordal case.

For $\kappa \geq 0$, set $\lambda(t)=\sqrt{\kappa} B_{t}$, where $B_{t}$ is standard Brownian motion. Then chordal $\mathrm{SLE}_{\kappa}$ is the random family of conformal maps generated by $\lambda$, that is, the family of maps solving the following stochastic differential equation:

$$
\begin{aligned}
\frac{\partial}{\partial t} g_{t}(z) & =\frac{2}{g_{t}(z)-\sqrt{\kappa} B_{t}}, \\
g_{0}(z) & =z .
\end{aligned}
$$

For SLE, it is possible to define an almost surely continuous path $\gamma:[0, \infty) \rightarrow \overline{\mathbb{H}}$ such that the domains $G_{t}$ generated by $\lambda(t)=\sqrt{\kappa} B_{t}$ are the unbounded components of $\mathbb{H} \backslash \gamma[0, t]$ for every $t \geq 0$. See $[9]$ and, for the case $\kappa=8$, [6]. The random curve $\gamma$ is referred to as the "trace" and is often considered to be the process $\mathrm{SLE}_{\kappa}$ rather than the random family of conformal maps. S. Rohde and O. Schramm 9 have shown the following classification for the trace curves:

(1) For $\kappa \in[0,4], \gamma(t)$ is almost surely a simple path contained in $\mathbb{H} \cup\{0\}$.

(2) For $\kappa \in(4,8), \gamma(t)$ is almost surely a non-simple path.

(3) For $\kappa \in[8, \infty), \gamma(t)$ is almost surely a space-filling curve.

This classification is roughly illustrated in Figure 3, Let us note that more accurate pictures would not look quite so tame.

There has been much progress recently in studying SLE. See [4] for a short survey paper with an extensive bibliography. 

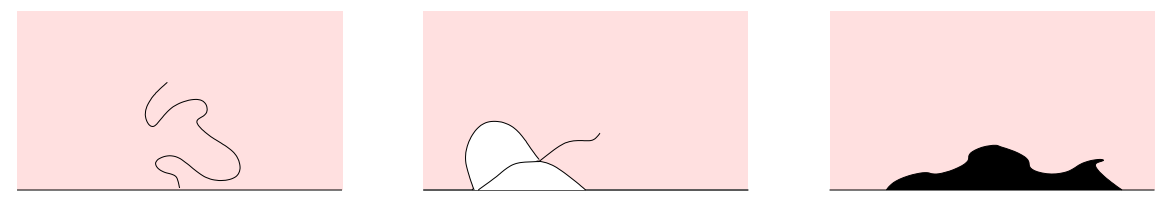

Figure 3. The $\mathrm{SLE}_{\kappa}$ trace is a simple curve, a non-simple curve, or a space-filling curve depending on the value of $\kappa$. The colored region is the domain $G_{t}$.

\section{NATURAL PARAMETRIZATIONS OF THE TRACE}

Let $\gamma$ be the (random) trace associated with $\operatorname{SLE}_{\kappa}$. We usually consider $\gamma$ not as a set, but as a curve with a specific parametrization, namely the one given by $\gamma(t)=$ $g_{t}^{-1}(\lambda(t))=g_{t}^{-1}\left(\sqrt{\kappa} B_{t}\right)$, which is well-defined since $g_{t}^{-1}$ extends continuously to the real line. We will refer to this as parametrizing by capacity. (We give it this name since near infinity $g_{t}(z)=z+\frac{2 t}{z}+O\left(\frac{1}{z^{2}}\right)$ and the $2 t$ term represents a kind of capacity of the hull $K_{t}$.) There is a second natural way to parametrize the trace. For this method, we run the Loewner equation until time $T$ and then use the map $g_{T}^{-1}$, extended continuously to the real line, to parametrize the portion of $\gamma$ seen at time $T$ by setting $\gamma(x)=g_{T}^{-1}(x)$, for appropriate values of $x$. This we call parametrizing by $g_{T}^{-1}$. Notice that this second way of parametrizing $\gamma$ is only valid for $\kappa \leq 4$. When $\kappa>4$, this gives a parametrization of the outer boundary of the hull. Both of these natural parametrizations have some Hölder regularity, which follows from computations done in 9 . In particular, the work of S. Rohde and O. Schramm gives that $\gamma$ is Hölder continuous with exponent $\hat{\sigma}-\epsilon$ under the first parametrization, where $\epsilon>0$ and

$$
\hat{\sigma}(\kappa)= \begin{cases}\frac{1}{2} \frac{(\kappa-8)^{2}}{(\kappa+8)^{2}}, & 0 \leq \kappa \leq 8, \\ 1-\frac{1}{2} \frac{\kappa}{\kappa+12-4 \sqrt{\kappa+8}}, & 8<\kappa .\end{cases}
$$

In addition, their work gives that $\gamma$ (or the outer boundary when $\kappa>4$ ) is Hölder continuous with exponent $\hat{\eta}-\epsilon$ when parametrized by $g_{T}^{-1}$, where $\epsilon>0$ and

$$
\hat{\eta}(\kappa)= \begin{cases}1-\frac{4}{3 \kappa+8-2 \sqrt{2 \kappa(\kappa+4)}}, & 0 \leq \kappa \leq 4+4 \sqrt{5}, \\ \frac{1}{2}-\frac{4}{\kappa}, & 4+4 \sqrt{5}<\kappa \leq 6+2 \sqrt{17}, \\ 2-\frac{(\kappa+4)^{2}}{\kappa^{2}+12 \kappa+16-2 \sqrt{2 \kappa(\kappa+2)(\kappa+8)}}, & 6+2 \sqrt{17}<\kappa .\end{cases}
$$

Notice that $\hat{\eta}(\kappa) \nearrow 1$ and $\hat{\sigma}(\kappa) \nearrow \frac{1}{2}$ as $\kappa \rightarrow \infty$. The latter is surprising since our intuition is that while the outer boundary should become smoother as $\kappa$ becomes large, the trace should continue to become wilder. Notice also that for both of these parametrizations, the Hölder exponent is $\frac{1}{2}-\epsilon$ when $\kappa=0$. This is no surprise, since from our calculations we know that $g_{t}^{-1}(z)=\sqrt{z^{2}-4 t}$ for $\kappa=0$. Thus the capacity parametrization is $\gamma(t)=i 2 \sqrt{t}$, and the second parametrization is $\gamma(x)=i \sqrt{4 T-x^{2}}$ for $T$ fixed and $x \in[0,2 \sqrt{T}]$. However, we also know that when $\kappa=0$, the curve $\gamma$ is as smooth as possible - it is a straight line, which makes an angle of $\frac{\pi}{2}$ with the real line, as shown in Figure 2, This angle is the reason that the Hölder exponent is $\frac{1}{2}$, rather than an exponent of 1 which better reflects the regularity of the curve. 


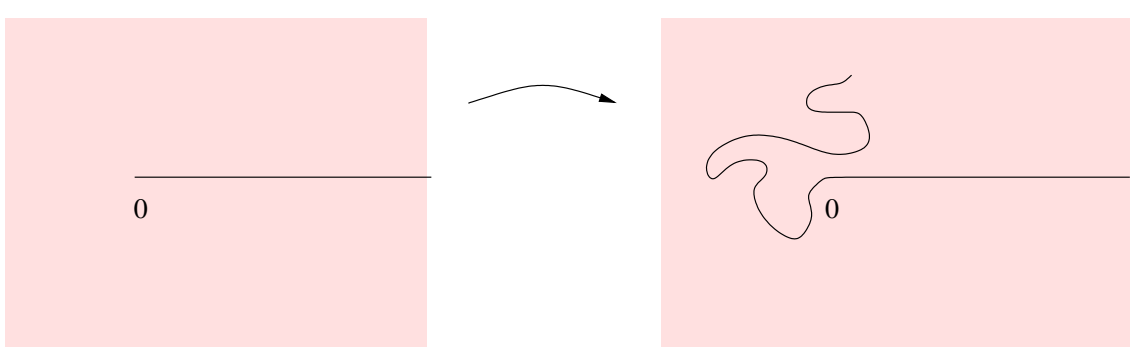

Figure 4 . The function $h_{t}$ is a conformal map from $\mathbb{C} \backslash[0, \infty)$ into $\mathbb{C} \backslash[0, \infty)$. In the case that $\kappa \leq 4, h_{t}$ maps onto a slit plane roughly like the one pictured above.

This suggests that either we should find another way to formulate our question about the regularity of the trace or we should look to find a different parametrization which has a larger Hölder exponent. Working with the first tactic, we consider the function

$$
h_{t}(z):=\left(f_{t}(\sqrt{z})-\lambda(t)\right)^{2},
$$

which is a conformal map from $\mathbb{C} \backslash[0, \infty)$ into $\mathbb{C} \backslash[0, \infty)$, as illustrated in Figure 4, By $f_{t}$ we mean the conformal map from $\mathbb{H}$ into $\mathbb{H}$ satisfying (2), what we refer to as the backward Loewner equation, with $\xi(t)=\lambda(t)=\sqrt{\kappa} B_{t}$. Also, recall from [9] that for fixed $t, f_{t}(z)-\lambda(t)$ is equal in distribution to $g_{t}^{-1}(z+\lambda(t))$.

We let $\gamma_{2}$ denote the trace in this new setting. As we discuss in the final section of this paper, to parametrize $\gamma_{2}$ by capacity, we set $\gamma_{2}(t)=\left(g_{t}^{-1}(\lambda(t))\right)^{2}$. In the penultimate section, we discuss parametrizing $\gamma_{2}$ using $h_{T}$, which means setting $\gamma_{2}(x)=\lim _{y \rightarrow 0^{+}} h_{T}(x+i y)$ for $T$ fixed and $x \in\left[0, x_{T}\right]$. Again this latter parametrization is valid only for $\kappa \leq 4$. When $\kappa=0$, both natural parametrizations of $\gamma_{2}$ are clearly Hölder continuous with exponent 1, as desired. In this new setting, we have gotten rid of the former problem of the trace making an angle of $\frac{\pi}{2}$ with the real line. In the remainder of this paper, we analyze the function $h_{t}$ in order to determine bounds on the Hölder exponent of $\gamma_{2}$ under each of these two natural parametrizations, making much use of the techniques of $[9]$.

\section{LEMMAS}

For $\alpha>0$, we would like to understand $E\left[\left|h_{t}^{\prime}(z)\right|^{\alpha}\right]$ as a function of $t \in[0,1]$ and $z \in \mathbb{H}$. We follow the methods in [9]. Recall that

$$
d\left(f_{t}(\sqrt{z})-\lambda(t)\right)=\frac{-2}{f_{t}(\sqrt{z})-\lambda(t)} d t-\sqrt{\kappa} d B_{t} .
$$

Applying Itô's formula and differentiating in $z$ gives

$$
d h_{t}^{\prime}(z)=-\frac{\sqrt{\kappa}}{\sqrt{h_{t}(z)}} h_{t}^{\prime}(z) d B_{t} .
$$

Another application of Itô's formula yields

$$
d \log \left(h_{t}^{\prime}(z)\right)=-\frac{\kappa}{2} \frac{1}{h_{t}(z)} d t-\sqrt{\kappa} \frac{1}{\sqrt{h_{t}(z)}} d B_{t} .
$$


Set $X_{t}:=\operatorname{Re} \sqrt{h_{t}(z)}=\operatorname{Re}\left(f_{t}(\sqrt{z})-\lambda(t)\right)$ and set $Y_{t}:=\operatorname{Im} \sqrt{h_{t}(z)}=\operatorname{Im} f_{t}(\sqrt{z})$, which satisfy

$$
\begin{aligned}
d X_{t} & =\frac{-2 X_{t}}{X_{t}^{2}+Y_{t}^{2}} d t-\sqrt{\kappa} d B_{t}, \\
d Y_{t} & =\frac{2 Y_{t}}{X_{t}^{2}+Y_{t}^{2}} d t
\end{aligned}
$$

Therefore we have

$$
\begin{aligned}
d \log \left|h_{t}^{\prime}(z)\right| & =\operatorname{Re} d \log \left(h_{t}^{\prime}(z)\right) \\
& =-\frac{\kappa}{2} \frac{X_{t}^{2}-Y_{t}^{2}}{\left(X_{t}^{2}+Y_{t}^{2}\right)^{2}} d t-\sqrt{\kappa} \frac{X_{t}}{X_{t}^{2}+Y_{t}^{2}} d B_{t} .
\end{aligned}
$$

As in 9, we would like to change both time and space variables. Set $u:=$ $\log \left(\frac{Y_{t}}{Y_{0}}\right)$ and set $W_{t}:=\frac{X_{t}}{Y_{t}}$. Notice that

$$
d W_{t}=\frac{-4 W_{t}}{X_{t}^{2}+Y_{t}^{2}} d t-\sqrt{\kappa} \frac{1}{Y_{t}} d B_{t} .
$$

Using that $\frac{\partial u}{\partial t}=\frac{2}{X_{t}^{2}+Y_{t}^{2}}$, changing to " $u$-time" gives

$$
d W_{u}=-2 W_{u} d u-\sqrt{\frac{\kappa}{2}} \sqrt{W_{u}^{2}+1} d \hat{B}_{u},
$$

and

$$
d \log \left|h_{u}^{\prime}(z)\right|=-\frac{\kappa}{4} \frac{W_{u}^{2}-1}{W_{u}^{2}+1} d u-\sqrt{\frac{\kappa}{2}} \frac{W_{u}}{\sqrt{W_{u}^{2}+1}} d \hat{B}_{u},
$$

where by $h_{u}^{\prime}(z)$ we mean $h_{t(u)}^{\prime}(z)$ and $\hat{B}_{u}$ is a Brownian motion with respect to " $u$-time." To simplify notation, we will refer to this simply as $B_{u}$.

Now for $\alpha>0$,

$$
\begin{aligned}
E\left[\left|h_{u}^{\prime}(z)\right|^{\alpha}\right] & =E\left[\exp \left(\alpha \int_{0}^{u} d \log \left|h_{u}^{\prime}(z)\right|\right)\right] \\
& =E\left[\exp \left(\int_{0}^{u}-\alpha \frac{\kappa}{4} \frac{W_{u}^{2}-1}{W_{u}^{2}+1} d u+\int_{0}^{u}-\alpha \sqrt{\frac{\kappa}{2}} \frac{W_{u}}{\sqrt{W_{u}^{2}+1}} d B_{u}\right)\right] .
\end{aligned}
$$

We find ourselves in a more complicated situation than in 9 because of the integral with respect to $B_{u}$ in the above expression. However, making use of the Girsanov transformation (discussed in [8]) takes care of this complication. Let

$$
M_{u}:=\exp \left(\int_{0}^{u}-\alpha \sqrt{\frac{\kappa}{2}} \frac{W_{u}}{\sqrt{W_{u}^{2}+1}} d B_{u}-\frac{1}{2} \int_{0}^{u} \alpha^{2} \frac{\kappa}{2} \frac{W_{u}^{2}}{W_{u}^{2}+1} d u\right),
$$

and notice that Novikov's condition is satisfied, that is,

$$
E\left[\exp \left(\frac{1}{2} \int_{0}^{u_{1}} \alpha^{2} \frac{\kappa}{2} \frac{W_{u}^{2}}{W_{u}^{2}+1} d u\right)\right]<\infty
$$

since $\frac{W_{u}^{2}}{W_{u}^{2}+1} \leq 1$. Thus, applying the Girsanov transformation gives that

$$
\tilde{B}_{u}:=\int_{0}^{u} \alpha \sqrt{\frac{\kappa}{2}} \frac{W_{u}}{\sqrt{W_{u}^{2}+1}} d u+B_{u}
$$


is a Brownian motion under $\tilde{P}$, where $d \tilde{P}=M_{u} d P$. Note that

$$
d W_{u}=\left(\frac{\kappa}{2} \alpha-2\right) W_{u} d u-\sqrt{\frac{\kappa}{2}} \sqrt{W_{u}^{2}+1} d \tilde{B}_{u},
$$

and further we have

$$
\begin{aligned}
E\left[\left|h_{u}^{\prime}(z)\right|^{\alpha}\right] & =E\left[\exp \left(\int_{0}^{u}-\alpha \frac{\kappa}{4} \frac{W_{u}^{2}-1}{W_{u}^{2}+1} d u+\int_{0}^{u}-\alpha \sqrt{\frac{\kappa}{2}} \frac{W_{u}}{\sqrt{W_{u}^{2}+1}} d B_{u}\right)\right] \\
& =\tilde{E}\left[\exp \left(\int_{0}^{u}-\alpha \frac{\kappa}{4} \frac{W_{u}^{2}-1}{W_{u}^{2}+1} d u+\int_{0}^{u} \alpha^{2} \frac{\kappa}{4} \frac{W_{u}^{2}}{W_{u}^{2}+1} d u\right)\right] \\
& =\tilde{E}\left[\exp \left(\int_{0}^{u} \frac{\kappa}{4} \alpha \frac{(\alpha-1) W_{u}^{2}+1}{W_{u}^{2}+1} d u\right)\right] \\
& =: v(w, u),
\end{aligned}
$$

where $w=W_{0}$. By Feynman-Kac (see [8]), $v$ is the solution to the following initial value problem:

$$
\begin{gathered}
\frac{\partial v}{\partial u}=\left(\frac{\kappa}{2} \alpha-2\right) w \frac{\partial v}{\partial w}+\frac{\kappa}{4}\left(w^{2}+1\right) \frac{\partial^{2} v}{\partial w^{2}}+\frac{\kappa}{4} \alpha \frac{(\alpha-1) w^{2}+1}{w^{2}+1} v \\
v(w, 0)=1
\end{gathered}
$$

Ignoring the initial condition for a moment, we have that $\left(1+w^{2}\right)^{b} \mathrm{e}^{L u}$ satisfies (3) when the following two conditions hold:

$$
\begin{gathered}
L=\frac{\kappa}{2} b+\frac{\kappa}{4} \alpha, \\
-b^{2}+\left(\frac{4}{k}+1-\alpha\right) b+\frac{\alpha}{2}-\frac{\alpha^{2}}{4}=0 .
\end{gathered}
$$

If we had concerned ourselves instead with $E\left[\left(1+W_{u}^{2}\right)^{b}\left|h_{u}^{\prime}(z)\right|^{\alpha}\right]=: \hat{v}(w, u)$, our previous arguments would show that

$$
\hat{v}(w, u)=\tilde{E}\left[\left(1+W_{u}^{2}\right)^{b} \exp \left(\int_{0}^{u} \frac{\kappa}{4} \alpha \frac{(\alpha-1) W_{u}^{2}+1}{W_{u}^{2}+1} d u\right)\right] .
$$

Applying Feynman-Kac in this slightly revised situation gives that $\hat{v}$ is also a solution to (3) but with initial condition changed to $\hat{v}(w, 0)=\left(1+w^{2}\right)^{b}$. Therefore, with $b$ and $L$ as in (4), we would expect that

$$
E\left[\left(1+W_{u}^{2}\right)^{b}\left|h_{u}^{\prime}(z)\right|^{\alpha}\right]=\left(1+w^{2}\right)^{b} \mathrm{e}^{L u} .
$$

This is the content of the following lemma.

Lemma 1. If $\alpha>0$ and $\alpha, b$, and $L$ are related by (4), then

$$
E\left[\left(1+W_{u}^{2}\right)^{b}\left|h_{u}^{\prime}(z)\right|^{\alpha}\right]=\left(1+w^{2}\right)^{b} \mathrm{e}^{L u} .
$$

Proof. Let $D$ be the differential operator defined by

$$
D F:=\frac{\partial F}{\partial u}-\left(\frac{\kappa}{2} \alpha-2\right) w \frac{\partial F}{\partial w}-\frac{\kappa}{4}\left(w^{2}+1\right) \frac{\partial^{2} F}{\partial w^{2}}-\frac{\kappa}{4} \alpha \frac{(\alpha-1) w^{2}+1}{w^{2}+1} F .
$$


Then $D \hat{v}=0$ for $\hat{v}(w, u)=E\left[\left(1+W_{u}^{2}\right)^{b}\left|h_{u}^{\prime}(z)\right|^{\alpha}\right]$ as discussed above. Let $\epsilon>0$ and set

$$
F_{\epsilon}(w, u):=(1+\epsilon) \mathrm{e}^{(L+\epsilon) u}\left(1+w^{2}\right)^{b+\epsilon^{2}} .
$$

Then $\hat{v}(w, 0)<F_{\epsilon}(w, 0)$ and $D F_{\epsilon}>0$ for $\epsilon$ small enough.

We claim that for $u \in\left[0, C_{0}\right]$ and $|w|$ large enough, $\hat{v}(w, u)<F_{\epsilon}(w, u)$. After showing that this claim implies

$$
E\left[\left(1+W_{u}^{2}\right)^{b}\left|h_{u}^{\prime}(z)\right|^{\alpha}\right] \leq\left(1+w^{2}\right)^{b} \mathrm{e}^{L u},
$$

we will verify the claim. Suppose that $\hat{v}(w, u) \geq F_{\epsilon}(w, u)$ for some point $(w, u)$ in $\mathbb{R} \times[0, \infty)$. Then, supposing the claim holds, there must be a point $\left(w_{0}, u_{0}\right)$ with $u_{0}>0$ minimal and $\hat{v}\left(w_{0}, u_{0}\right)=F_{\epsilon}\left(w_{o}, u_{0}\right)$. Let us consider what must happen at this point: $F_{\epsilon}-\hat{v}$ must have a local minimum in the $w$-direction and must be non-increasing in the $u$-direction. In addition, we have that $D\left(F_{\epsilon}-\hat{v}\right)\left(w_{0}, u_{0}\right)>0$. These conditions, however, lead to a contraction. Therefore $\hat{v}(w, u)<F_{\epsilon}(w, u)$ on $\mathbb{R} \times[0, \infty)$. Letting $\epsilon$ go to 0 gives that

$$
E\left[\left(1+W_{u}^{2}\right)^{b}\left|h_{u}^{\prime}(z)\right|^{\alpha}\right] \leq\left(1+w^{2}\right)^{b} \mathrm{e}^{L u} .
$$

We now show that for $u \in\left[0, C_{0}\right]$ and $|w|$ large enough, $\hat{v}(w, u)<F_{\epsilon}(w, u)$. Notice that for $u \in\left[0, C_{0}\right]$,

$$
\begin{aligned}
\frac{\hat{v}(w, u)}{F_{\epsilon}(w, u)} & =\frac{\tilde{E}\left[\left(1+W_{u}^{2}\right)^{b} \exp \left(\int_{0}^{u} \frac{\kappa}{4} \alpha \frac{(\alpha-1) W_{u}^{2}+1}{W_{u}^{2}+1} d u\right)\right]}{(1+\epsilon) \mathrm{e}^{(L+\epsilon) u}\left(1+w^{2}\right)^{b+\epsilon^{2}}} \\
& \leq C\left(1+w^{2}\right)^{-\epsilon^{2}} \tilde{E}\left[\left(\frac{1+W_{u}^{2}}{1+w^{2}}\right)^{b}\right],
\end{aligned}
$$

and so the claim follows if we can bound the expectation term in the line above. We do this by utilizing our tools of Itô calculus and the Girsanov transformation again. First, we compute that

$$
d \log \left(\frac{1+W_{u}^{2}}{1+w^{2}}\right)=\frac{\kappa}{2} \frac{1+(2 \alpha-8 / \kappa-1) W_{u}^{2}}{1+W_{u}^{2}} d u-\sqrt{2 \kappa} \frac{W_{u}}{\sqrt{1+W_{u}^{2}}} d \tilde{B}_{u} .
$$

Then for $u \in\left[0, C_{0}\right]$,

$$
\begin{aligned}
\tilde{E} & {\left[\left(\frac{1+W_{u}^{2}}{1+w^{2}}\right)^{b}\right] } \\
& =\tilde{E}\left[\exp \left(\int_{0}^{u} \frac{\kappa b}{2} \frac{1+(2 \alpha-8 / \kappa-1) W_{u}^{2}}{1+W_{u}^{2}} d u-\int_{0}^{u} b \sqrt{2 \kappa} \frac{W_{u}}{\sqrt{1+W_{u}^{2}}} d \tilde{B}_{u}\right)\right] \\
& =E^{*}\left[\exp \left(\int_{0}^{u} \frac{\kappa b}{2} \frac{1+(2 \alpha-8 / \kappa-1) W_{u}^{2}}{1+W_{u}^{2}} d u+\int_{0}^{u} \kappa b^{2} \frac{W_{u}^{2}}{1+W_{u}^{2}} d u\right)\right] \\
& =E^{*}\left[\exp \left(\int_{0}^{u} \frac{\kappa b}{2} \frac{1+(2 \alpha-8 / \kappa-1+2 b) W_{u}^{2}}{1+W_{u}^{2}} d u\right)\right],
\end{aligned}
$$


where we used the Girsanov transformation with Radon-Nikodym derivative

$$
\frac{d P^{*}}{d \tilde{P}}=\exp \left(\int_{0}^{u}-b \sqrt{2 \kappa} \frac{W_{u}}{\sqrt{W_{u}^{2}+1}} d \tilde{B}_{u}-\frac{1}{2} \int_{0}^{u} 2 \kappa b^{2} \frac{W_{u}^{2}}{W_{u}^{2}+1} d u\right) .
$$

Since the function $\frac{1+c x}{1+x}$ is monotone in $x$, for $x \in[0, \infty)$, we have that $\frac{1+c W_{u}^{2}}{1+W_{u}^{2}}$ is bounded between 1 and $c$. Therefore

$$
\tilde{E}\left[\left(\frac{1+W_{u}^{2}}{1+w^{2}}\right)^{b}\right] \leq \mathrm{e}^{C u},
$$

and hence this expectation is bounded since $u \leq C_{0}$.

A similar argument gives that

$$
E\left[\left(1+W_{u}^{2}\right)^{b}\left|h_{u}^{\prime}(z)\right|^{\alpha}\right] \geq\left(1+w^{2}\right)^{b} \mathrm{e}^{L u},
$$

completing the proof.

This immediately implies the following.

Corollary 1. If $\alpha>0$ and $b \geq 0$, then

$$
E\left[\left|h_{u}^{\prime}(z)\right|^{\alpha}\right] \leq\left(1+\frac{x^{2}}{y^{2}}\right)^{b} \mathrm{e}^{L u}
$$

where $\alpha, b$, and $L$ are related by (4).

For this to be useful, it remains to change time from $u$ back to $t$.

Lemma 2. Suppose $t \leq 1, z \in \mathbb{C} \backslash[0, \infty)$ with $|z| \leq R$, and $\sqrt{z}=x+i y$. Then

$$
P\left[\left|h_{t}^{\prime}(z)\right|>\delta\right] \leq C_{\kappa, \alpha} M^{\alpha} \delta^{-\alpha} y^{-L}\left(1+\frac{x^{2}}{y^{2}}\right)^{b}+C_{\kappa, \mu} M^{-\mu} \log \left(\frac{C}{y}\right)
$$

for $\delta, \alpha, \mu, M$ positive and $L, b$ non-negative with $L=\frac{\kappa}{2} b+\frac{\kappa}{4} \alpha$ and $-b^{2}+$ $\left(\frac{4}{k}+1-\alpha\right) b+\frac{\alpha}{2}-\frac{\alpha^{2}}{4}=0$.

Proof. Recall that $u=\log \left(\frac{Y_{t}}{y}\right)$ and that, in order to simplify notation, we write $h_{u}$ for $h_{t(u)}$. In what follows, we will decompose " $u$-time" into intervals $[n, n+1)$, and hence the notation $h_{n}$ means $h_{t(n)}$. Note that for $|z| \leq R$ and $t \leq 1, Y_{t}$ is bounded by a universal constant $C$. (In particular, $Y_{t} \leq \sqrt{y^{2}+4 t}$.) So, $u \leq N$, where $N$ is the smallest integer greater than $\log \left(C y^{-1}\right)$. For $M>0$, set $M_{n}=\{\omega: u \in[n, n+1)$ and $\left.\left|h_{u}^{\prime}(z)\right|>M\left|h_{n}^{\prime}(z)\right|\right\}$. Notice that if $\omega$ satisfies that $\left|h_{u}^{\prime}(z)\right|>\delta, u \in[n, n+1)$ and $\omega \notin M_{n}$, then $\left|h_{n}^{\prime}(z)\right|>\frac{\delta}{M}$. Thus for $\delta>0$,

$$
\begin{aligned}
P\left[\left|h_{t}^{\prime}(z)\right|>\delta\right] & =\sum_{n=0}^{N} P\left[\left|h_{u}^{\prime}(z)\right|>\delta, u \in[n, n+1)\right] \\
& \leq \sum_{n=0}^{N} P\left[\left|h_{n}^{\prime}(z)\right|>\frac{\delta}{M}\right]+\sum_{n=0}^{N} P\left[M_{n}\right] .
\end{aligned}
$$


Let us consider the first sum above. For $\alpha>0$ and $b \geq 0$, we have

$$
\begin{aligned}
\sum_{n=0}^{N} P\left[\left|h_{n}^{\prime}(z)\right|>\frac{\delta}{M}\right] & =\sum_{n=0}^{N} P\left[\left(M \delta^{-1}\left|h_{n}^{\prime}(z)\right|\right)^{\alpha}>1\right] \\
& \leq M^{\alpha} \delta^{-\alpha} \sum_{n=0}^{N} E\left[\left|h_{n}^{\prime}(z)\right|^{\alpha}\right] \\
& \leq M^{\alpha} \delta^{-\alpha} \sum_{n=0}^{N}\left(1+\frac{x^{2}}{y^{2}}\right)^{b} \mathrm{e}^{L n}
\end{aligned}
$$

where Corollary 1 gives the last inequality. Recall that $L=\frac{\kappa}{2} b+\frac{\kappa}{4} \alpha$, and so $L$ is positive. Therefore $\sum_{n=0}^{N} \mathrm{e}^{L n} \leq C_{\kappa, \alpha} y^{-L}$, and we have that

$$
\sum_{n=0}^{N} P\left[\left|h_{n}^{\prime}(z)\right|>\frac{\delta}{M}\right] \leq C_{\kappa, \alpha} M^{\alpha} \delta^{-\alpha} y^{-L}\left(1+\frac{x^{2}}{y^{2}}\right)^{b}
$$

as desired.

It remains to show that $\sum_{n=0}^{N} P\left[M_{n}\right] \leq C_{\kappa, \mu} M^{-\mu} \log \left(\frac{C}{y}\right)$. Define $U_{n}$ to be the event $\{\omega: u \in[n, n+1)\}$. Then for $\mu>0$,

$$
\begin{aligned}
& P\left[M_{n}\right] \\
& =P\left[\frac{\left|h_{u}^{\prime}(z)\right|}{\left|h_{n}^{\prime}(z)\right|}>M ; U_{n}\right] \\
& =P\left[\exp \left(\int_{n}^{u}-\frac{\kappa}{4} \frac{W_{u}^{2}-1}{W_{u}^{2}+1} d u+\int_{n}^{u}-\sqrt{\frac{\kappa}{2}} \frac{W_{u}}{\sqrt{W_{u}^{2}+1}} d B_{u}\right)>M ; U_{n}\right] \\
& =P\left[M^{-\mu} \exp \left(\int_{n}^{u}-\mu \frac{\kappa}{4} \frac{W_{u}^{2}-1}{W_{u}^{2}+1} d u+\int_{n}^{u}-\mu \sqrt{\frac{\kappa}{2}} \frac{W_{u}}{\sqrt{W_{u}^{2}+1}} d B_{u}\right)>1 ; U_{n}\right] \\
& \leq E\left[M^{-\mu} \exp \left(\int_{n}^{u}-\mu \frac{\kappa}{4} \frac{W_{u}^{2}-1}{W_{u}^{2}+1} d u+\int_{n}^{u}-\mu \sqrt{\frac{\kappa}{2}} \frac{W_{u}}{\sqrt{W_{u}^{2}+1}} d B_{u}\right) 1_{U_{n}}\right] \\
& =M^{-\mu} E^{* *}\left[\exp \left(\int_{n}^{u} \frac{\kappa}{4} \mu \frac{(\mu-1) W_{u}^{2}+1}{W_{u}^{2}+1} d u\right) 1_{U_{n}}\right] .
\end{aligned}
$$

The last equality results from applying the Girsanov transformation with the Radon-Nykodym derivative

$$
\frac{d P^{* *}}{d P}=\exp \left(\int_{n}^{u}-\mu \sqrt{\frac{\kappa}{2}} \frac{W_{u}}{\sqrt{W_{u}^{2}+1}} d B_{u}-\frac{1}{2} \int_{n}^{u} \mu^{2} \frac{\kappa}{2} \frac{W_{u}^{2}}{W_{u}^{2}+1} d u\right),
$$

for $u \in[n, n+1]$. Note that $\frac{(\mu-1) x+1}{x+1}$ is increasing for $\mu>2$, constant for $\mu=2$ and decreasing for $0<\mu<2$. Thus, $P\left[M_{n}\right] \leq C_{\kappa, \mu} M^{-\mu}$ where $C_{\kappa, \mu}=$ $\max \left\{\exp \left(\frac{\kappa}{4} \mu\right), \exp \left(\frac{\kappa}{4} \mu(\mu-1)\right)\right\}$, and so

$$
\sum_{n=0}^{N} P\left[M_{n}\right] \leq C_{\kappa, \mu} M^{-\mu} \log \left(\frac{C}{y}\right) .
$$

We also need a version of Lemma 2 for the case when $b<0$. 
Lemma 3. Suppose $t \leq 1, z \in \mathbb{C} \backslash[0, \infty)$ with $|z| \leq R$, and $\sqrt{z}=x+i y$. Then

$$
\begin{gathered}
P\left[\left|h_{t}^{\prime}(z)\right|>\delta\right] \leq C_{\kappa, \alpha} M^{\alpha} \delta^{-\alpha} D^{-2 b} y^{-L}\left(1+\frac{x^{2}}{y^{2}}\right)^{b}+C_{\kappa, \mu} M^{-\mu} \log \left(\frac{C}{y}\right) \\
+2 \mathrm{e}^{-D^{2} / 2 \kappa},
\end{gathered}
$$

for $\delta, \alpha, \mu, M$ positive, $D \geq R \vee 1, b<0$, and $L$ with $L=\frac{\kappa}{2} b+\frac{\kappa}{4} \alpha$ and $-b^{2}+$ $\left(\frac{4}{k}+1-\alpha\right) b+\frac{\alpha}{2}-\frac{\alpha^{2}}{4}=0$.

Proof. Let $b<0$, and let $N$ and $M_{n}$ be defined as in the proof of the previous lemma, and recall that $\sum_{n=0}^{N} P\left[M_{n}\right] \leq C_{\kappa, \mu} M^{-\mu} \log \left(\frac{C}{y}\right)$. By proceeding as before, we have that

$$
P\left[\left|h_{t}^{\prime}(z)\right|>\delta\right] \leq M^{\alpha} \delta^{-\alpha} \sum_{n=0}^{N} E\left[\left|h_{n}^{\prime}(z)\right|^{\alpha}\right]+C_{\kappa, \mu} M^{-\mu} \log \left(\frac{C}{y}\right) .
$$

Consequently, we wish to have an upper bound for $E\left[\left|h_{n}^{\prime}(z)\right|^{\alpha}\right]$ in the case that $b<0$.

Recall that

$$
E\left[\left(1+W_{n}^{2}\right)^{b}\left|h_{n}^{\prime}(z)\right|^{\alpha}\right]=\left(1+w^{2}\right)^{b} \mathrm{e}^{L n} .
$$

Now if $|\lambda(s)| \leq D$ for all $s \in[0,1]$, then $\left|X_{s}\right|=\left|\operatorname{Re} f_{s}-\lambda(s)\right| \leq 2 D$, since Re $f_{s}$ always moves toward $\lambda(s)$. Thus assuming $|\lambda(s)| \leq D$ on $[0,1]$, we have

$$
\begin{aligned}
1+W_{n}^{2} & =Y_{n}^{-2}\left(Y_{n}^{2}+X_{n}^{2}\right) \\
& \leq C D^{2} y^{-2} \mathrm{e}^{-2 n} .
\end{aligned}
$$

The inequality follows from the facts that $Y_{n}=y \mathrm{e}^{n}$ and $Y_{s} \leq \sqrt{R^{2}+4} \leq C D$ for $s \in[0,1]$. Thus

$$
\begin{aligned}
E\left[\left|h_{n}^{\prime}(z)\right|^{\alpha}\right] & \leq C D^{-2 b} y^{2 b} \mathrm{e}^{2 b n} E\left[\left(1+W_{n}^{2}\right)^{b}\left|h_{n}^{\prime}(z)\right|^{\alpha}\right] \\
& =C D^{-2 b} y^{2 b}\left(1+w^{2}\right)^{b} \mathrm{e}^{(2 b+L) n} .
\end{aligned}
$$

Note that $2 b+L=2 b+\frac{\kappa}{4} \pm \frac{\kappa}{4} \sqrt{1+\frac{16}{\kappa} b} \geq 0$ for all allowable $b$, that is, for $b \in\left[-\frac{\kappa}{16}, 0\right]$, regardless of the choice of sign. Having $2 b+L \geq 0$ implies that

$$
\sum_{n=0}^{N} \mathrm{e}^{(2 b+L) n} \leq C y^{-2 b-L}
$$

and so

$$
\sum_{n=0}^{N} E\left[\left|h_{n}^{\prime}(z)\right|^{\alpha}\right] \leq C D^{-2 b} y^{-L}\left(1+w^{2}\right)^{b},
$$

under the assumption that $|\lambda(s)| \leq D$ on $[0,1]$. Therefore,

$$
P\left[\left|h_{t}^{\prime}(z)\right|>\delta\right] \leq C M^{\alpha} \delta^{-\alpha} D^{-2 b} y^{-L}\left(1+\frac{x^{2}}{y^{2}}\right)^{b}+C_{\kappa, \mu} M^{-\mu} \log \left(\frac{C}{y}\right)+P_{D},
$$

where $P_{D}=P[|\lambda(s)|>D$ for some $s \in[0,1]]$. 
We finish the proof by showing that $P_{D} \leq 2 \mathrm{e}^{-D^{2} / 2 \kappa}$ :

$$
\begin{aligned}
P_{D} & =P[|\lambda(s)|>D \text { for some } s \in[0,1]] \\
& \leq 2 P\left[B_{s}>\frac{D}{\sqrt{\kappa}} \text { for some } s \in[0,1]\right] \\
& =\frac{4}{\sqrt{2 \pi}} \int_{D / \sqrt{\kappa}}^{\infty} \exp \left(\frac{-x^{2}}{2}\right) d x \\
& \leq \frac{4}{\sqrt{2 \pi}} \exp \left(\frac{-D^{2}}{2 \kappa}\right) \int_{0}^{\infty} \exp \left(\frac{-x^{2}}{2}\right) d x \\
& =2 \exp \left(\frac{-D^{2}}{2 \kappa}\right) .
\end{aligned}
$$

\section{The Hölder CONTINUity of $h_{t}$}

Equipped with the technical lemmas of the last section, we are ready to prove the Hölder continuity of the function $h_{t}$ and of the trace $\gamma_{2}$. We first look at the parametrization of $\gamma_{2}$ obtained from the continuous extension of $h_{T}$ to the real line. More precisely, for $\kappa \leq 4$ we define

$$
\gamma_{2}(x)=\lim _{y \rightarrow 0^{+}} h_{T}(x+i y),
$$

for $T$ fixed and $x \in\left[0, x_{T}\right]$ where $\lim _{y \rightarrow 0^{+}} h_{T}\left(x_{T}+i y\right)=0$. The next theorem gives a lower bound on the optimal Hölder exponent for $h_{T}$, and from this we immediately obtain Theorem 2, Recall that a function $h$ being Hölder continuous in $\mathbb{H}$ with exponent $\eta$ means that for all bounded $A \subset \mathbb{H}$, there is a constant $C=C(A)$ so that $\left|h\left(z_{1}\right)-h\left(z_{2}\right)\right| \leq C\left|z_{1}-z_{2}\right|^{\eta}$ for $z_{1}, z_{2} \in A$. In our case, when showing that $h_{T}$ is Hölder continuous, the constant $C$ in question will depend on $A, T$, and $\omega$.

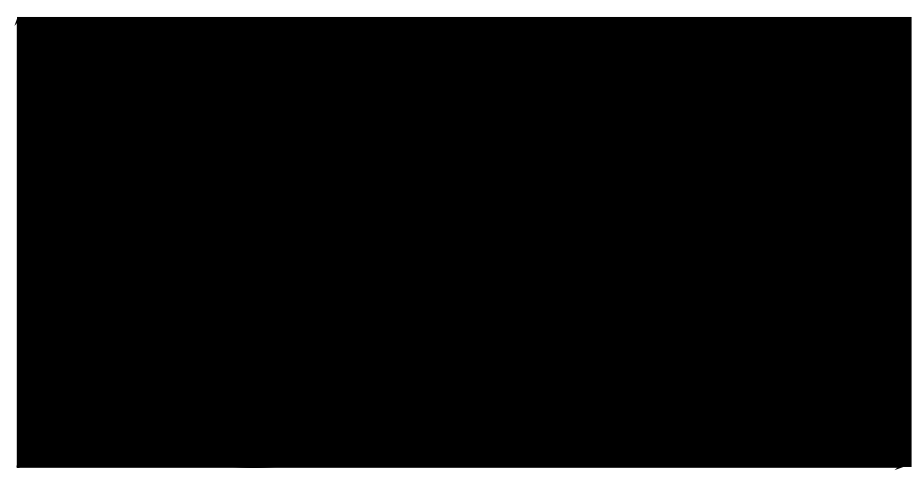

Figure 5 . The functions $\eta(\kappa) \geq \hat{\eta}(\kappa)$. For $\kappa<6+2 \sqrt{17}, \kappa \neq 4$, the inequality is strict.

Theorem 3. Let $\kappa \leq 33$. For all $t \geq 0$, a.s. $h_{t}$ is Hölder continuous in $\mathbb{H}$ with exponent $\eta-\epsilon$, where $\epsilon>0$ and

$$
\eta(\kappa)=2-\frac{(\kappa+4)^{2}}{\kappa^{2}+12 \kappa+16-2 \sqrt{2 \kappa(\kappa+2)(\kappa+8)}} .
$$


Notice that if $\kappa \geq 6+2 \sqrt{17}$, then $\eta(\kappa)$ agrees with the exponent $\hat{\eta}(\kappa)$ found in $[9$, and for $\kappa<6+2 \sqrt{17}, \kappa \neq 4$, this is an improvement over $\hat{\eta}(\kappa)$. This improvement is greatest for $\kappa$ near 0 , since $\eta(0)$ has the desired value of 1 , while $\hat{\eta}(0)=1 / 2$. See Figure 5 for the graphs of these two functions. The fact that $\eta(4)=\hat{\eta}(4)=0$ is inevitable, since when $\kappa=4$, the trace almost hits back on itself, meaning that $h_{t}$ and $f_{t}$ cannot be Hölder continuous.

Proof. From the scaling invariance of Brownian motion and SLE, we have that $h_{t}$ also satisfies a useful scaling property, that is, $r h_{t / r}(z / r)$ equals $h_{t}$ in distribution when $r>0$. Thus, it suffices to take $t \leq 1$ and show that $h_{t}$ is Hölder continuous on $A:=\left[-\frac{1}{2}, \frac{1}{2}\right] \times(0,1]$. We take a Whitney decomposition of $A$, as pictured in Figure 6. For each rectangle in this decomposition, we mark the midpoint of the top edge, and we let $\left\{z_{n, j}\right\}$ be the collection of these points. That is, $z_{n, j}=x_{n, j}+2^{-n} i \in A$ for $n \in \mathbb{N}$ and $j \in\left\{0,1, \cdots, 2^{n}-1\right\}$. When we apply Lemma 2 below, we will only need to know that there are $2^{n}$ of these points at height $2^{-n}$; however for the more difficult case, we will need to know that the particular value of $x_{n, j}$ is $-1 / 2+2^{-(n+1)}+j 2^{-n}$. We will show that

$$
\sum_{n, j} P\left[\left|h_{t}^{\prime}\left(z_{n, j}\right)\right|>2^{n(1-\eta)}\right]<\infty .
$$

This implies that $P\left[\left|h_{t}^{\prime}\left(z_{n, j}\right)\right|>2^{n(1-\eta)}\right.$ i.o. $]=0$ by the Borel-Cantelli Lemma. Therefore, by making use of the Koebe Distortion Theorem, we have that there exists $C>0$ so that a.s.

$$
\left|h_{t}^{\prime}(x+i y)\right| \leq \frac{C}{y^{1-\eta}}
$$

for $x+i y \in A$. This last statement implies that $h_{t}$ is Hölder continuous with exponent $\eta$ on $A$.

$\mathrm{z}(0,0)$

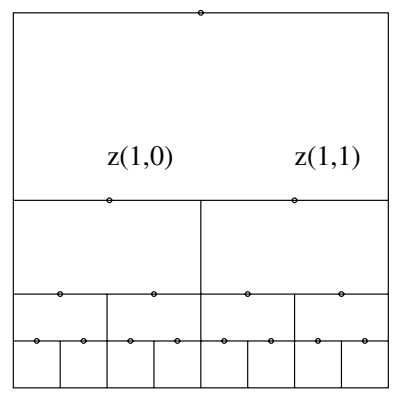

Figure 6. The Whitney decomposition of the unit square $\left[-\frac{1}{2}, \frac{1}{2}\right] \times[0,1]$, and the points $z_{n, j}=z(n, j)$.

To show (6), we wish to apply Lemma 2 and Lemma 3 . Fix $n$ for the moment. Let $\alpha, \mu, m$ be positive constants to be determined later, let $\delta=2^{n(1-\eta)}$, and let $M=2^{m n}$. Assume $b \geq 0$ and that $b$ and $L$ are given as before by $L=\frac{\kappa}{2} b+\frac{\kappa}{4} \alpha$ and $-b^{2}+\left(\frac{4}{k}+1-\alpha\right) b+\frac{\alpha}{2}-\frac{\alpha^{2}}{4}=0$. We also use that $\operatorname{Im} \sqrt{z_{n, j}} \geq \frac{1}{2} \operatorname{Im} z_{n, j}=\frac{1}{2} 2^{-n}$. Then applying Lemma 2 gives

$$
P\left[\left|h_{t}^{\prime}\left(z_{n, j}\right)\right|>2^{n(1-\eta)}\right] \leq C 2^{n(m \alpha-\alpha(1-\eta)+L+2 b)}+n C 2^{-n m \mu} .
$$


Since for each $n$ there are $2^{n}$ terms in the sum, we wish to show that

$$
\sum_{n} 2^{n(1+m \alpha-\alpha(1-\eta)+L+2 b)}+\sum_{n} n C 2^{n(1-m \mu)}<\infty .
$$

The first sum will be finite when

$$
1-\alpha(1-\eta)+L+2 b<0,
$$

for $m$ sufficiently small. Then by picking $\mu$ large enough, i.e. $\mu>\frac{1}{m}$, the second sum will also be finite. Equation (7) holds when

$$
\eta<1-\frac{1+2 b+L}{\alpha}=1-\frac{\kappa}{4}-\frac{1+2 b+(\kappa / 2) b}{1-2 b \pm \sqrt{1+(16 / \kappa) b}}
$$

where we have used

$$
\alpha=1-2 b \pm \sqrt{1+\frac{16}{\kappa} b} .
$$

For $b \in\left[-\frac{\kappa}{16}, 1+\frac{4}{\kappa}\right]$, the maximum of the right hand side of (8) occurs when

$$
b=\frac{4 \kappa^{2}+32 \kappa+32-\sqrt{2} \sqrt{\kappa^{5}+26 \kappa^{4}+240 \kappa^{3}+896 \kappa^{2}+1024 \kappa}}{4(\kappa+4)^{2}} .
$$

To obtain this maximum, we choose the positive sign in (8) for $\kappa \leq 4$ and the negative sign for $\kappa>4$. After some algebra we arrive at the desired values of $\eta$. It simply remains to show that we obtain the same values for $\eta$ in the case that $b$ is negative. We do this by making use of Lemma 3. (Note: when $b$ is given by (9), we have that $b \geq 0$ precisely when $\kappa \leq \sqrt{17}-3$. However the value of $\sqrt{17}-3$ is unimportant.)

Let $b$ be given as in (9) and assume $b<0$. We continue to use that $\alpha, b$, and $L$ are related by (4), that $M=2^{m n}$, and that $m, \alpha$, and $\mu$ are positive. Finally, let $D=n$. We also recall that $2 b+L \geq 0$. In our application of Lemma 3. we will need the following estimate:

$$
\begin{aligned}
& \sum_{j}\left(\operatorname{Im} \sqrt{z_{n, j}}\right)^{-(2 b+L)}\left|z_{n, j}\right|^{b} \\
& \quad=\sum_{j}\left(-x_{n, j}+\sqrt{x_{n, j}^{2}+2^{-2 n}}\right)^{-(b+L / 2)}\left(x_{n . j}^{2}+2^{-2 n}\right)^{b / 2} \\
& \leq C 2^{n L / 2} \sum_{j=1}^{2^{n}}\left(-j+\sqrt{j^{2}+4}\right)^{-(b+L / 2)}\left(j^{2}+4\right)^{b / 2} \\
& \leq C 2^{n L / 2} \sum_{j=1}^{2^{n}} j^{2 b+L / 2} \\
& \leq C 2^{n(L+2 b+1)} .
\end{aligned}
$$

The last inequality requires that $2 b+L / 2+1=2 b+\frac{\kappa}{8}-\frac{\kappa}{8} \sqrt{1+\frac{16}{\kappa} b}+1$ is positive, which is true for $\kappa<8\left(1+31^{1 / 3} 3^{-2 / 3} \operatorname{Re}(9+2 \sqrt{3} i)^{1 / 3}\right) \approx 33.5238$. Now when we 


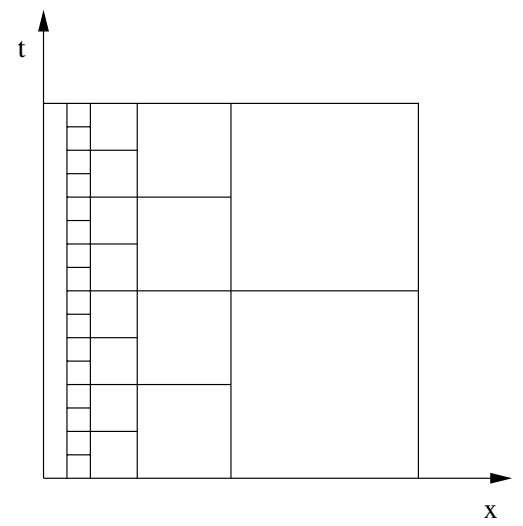

Figure 7 . The unit square $[0,1] \times[0,1]$ subdivided into squares $R_{j, k}$.

apply Lemma 3 we obtain

$$
\begin{aligned}
& \sum_{n, j} P\left[\left|h_{t}^{\prime}\left(z_{n, j}\right)\right|>2^{n(1-\eta)}\right] \\
& \quad \leq \sum_{n} n^{-2 b} 2^{n(1+m \alpha-\alpha(1-\eta)+L+2 b)}+\sum_{n} n C 2^{n(1-m \mu)}+\sum_{n} 2^{n-C n^{2}} .
\end{aligned}
$$

The third sum is clearly finite, and our consideration of the first two sums follows the arguments above.

\section{PARAMETRIZING THE TRACE BY CAPACITY}

Define $H(x, t):=\left(g_{t}^{-1}(i \sqrt{x}+\lambda(t))\right)^{2}$ on $[0, \infty) \times[0, \infty)$. We parametrize the trace by capacity by setting

$$
\gamma_{2}(t)=H(0, t)=\left(g^{-1}(\lambda(t))\right)^{2} .
$$

From Theorem 4 below, we obtain a lower bound for the Hölder exponent for this parametrization, proving Theorem 1 . Note that in this section, we will also use the notation $\hat{f}_{t}(z)=g_{t}^{-1}(z+\lambda(t))$.

Theorem 4. A.s. $H(x, t)$ is continuous on $[0, \infty) \times[0, \infty)$, and further, $H(0, t)$ is Hölder continuous with exponent $\sigma-\epsilon$, where $\epsilon>0$ and

$$
\sigma(\kappa)=1-\frac{\kappa}{2 \kappa+24-8 \sqrt{\kappa+8}} .
$$

Note that as with the previous parametrization, when $\kappa$ is small, we have obtained better Hölder exponents than in [9]. More specifically, for $\kappa<8, \sigma(\kappa)$ is an improvement over $\hat{\sigma}(\kappa)$, while for $\kappa \geq 8$, we have $\sigma(\kappa)=\hat{\sigma}(\kappa)$.

Proof. By scaling, it suffices to consider $H$ on $[0,1] \times[0,1]$. Decompose the square $[0,1] \times[0,1]$ as shown in Figure 7 , by setting

$$
R_{j, k}=\left[2^{-j}, 2^{-j+1}\right] \times\left[k 2^{-j},(k+1) 2^{-j}\right]
$$

for $j \geq 1$ and $0 \leq k \leq 2^{j}-1$. That is, $R_{j, k}$ is a square of sidelength $2^{-j}$, with the index $k$ referring to its vertical placement. 
Let $\sigma>0$. We wish to show that

$$
\sum_{j, k} P\left[\operatorname{diam}\left(H\left(R_{j, k}\right)\right)>2^{-j \sigma}\right]<\infty
$$

for any $\sigma<\sigma(\kappa)$ given above. Once we have shown (10), the Borel-Cantelli Lemma then implies that

$$
P\left[\operatorname{diam}\left(H\left(R_{j, k}\right)\right)>2^{-j \sigma} \text { i.o. }\right]=0,
$$

which in turn implies that there exists $C=C(\omega)$ so that a.s. $\operatorname{diam}\left(H\left(R_{j, k}\right)\right) \leq$ $C 2^{-j \sigma}$

Let $\left(x_{1}, t_{1}\right)$ and $\left(x_{2}, t_{2}\right)$ be points in $(0,1] \times(0,1]$, and let $j_{i}, k_{i}$ be the indices so that $\left(x_{i}, t_{i}\right) \in R_{j_{i}, k_{i}}$ for $i=1,2$. Consider the horizontal line $t=t_{1}$, and let $\left\{R_{j, k(1, j)}\right\}_{j \geq 1}$ be the collection of squares that intersect this line. In the case that the line intersects the boundary between two squares, simply choose one of the two to add to the collection. Similarly, we obtain $\left\{R_{j, k(2, j)}\right\}_{j \geq 1}$, a collection of squares that intersect the horizontal line $t=t_{2}$. Let $j_{*}$ be the largest $j$ such that the squares $R_{j, k(1, j)}$ and $R_{j, k(2, j)}$ are adjacent, and note that this implies that

$$
2^{-\left(j_{*}+1\right)} \leq\left|t_{1}-t_{2}\right| \leq 2^{-j_{*}+1} .
$$

Finally, set $j_{0}=\min \left\{j_{1}, j_{2}, j_{*}\right\}$. To control $\left|H\left(x_{1}, t_{1}\right)-H\left(x_{2}, t_{2}\right)\right|$, we will control how much $H$ can stretch the path made of straight line segments which connect the points $\left(x_{1}, t_{1}\right),\left(2^{-j_{0}}, t_{1}\right),\left(2^{-j_{0}}, t_{2}\right),\left(x_{2}, t_{2}\right)$ in this order. Thus, assuming equation (10) holds, we have

$$
\begin{aligned}
\left|H\left(x_{1}, t_{1}\right)-H\left(x_{2}, t_{2}\right)\right| & \leq \sum_{j \geq j_{0}} \operatorname{diam}\left(H\left(R_{j, k(1, j)}\right)\right)+\sum_{j \geq j_{0}} \operatorname{diam}\left(H\left(R_{j, k(2, j)}\right)\right) \\
& \leq C \sum_{j \geq j_{0}} 2^{-j \sigma} \\
& \leq C 2^{-j_{0} \sigma} .
\end{aligned}
$$

This gives that a.s. $H(x, t)$ is continuous on $[0,1] \times[0,1]$. Also, by letting $x_{1}$ and $x_{2}$ approach 0 , we have that a.s. $\left|H\left(0, t_{1}\right)-H\left(0, t_{2}\right)\right| \leq C\left|t_{1}-t_{2}\right|^{\sigma}$, and hence $H(0, t)$ is Hölder continuous with exponent $\sigma$ a.s. Therefore, we will have completed the proof once we have shown that equation (10) holds.

Now fix a square $R_{j, k}$. We recursively define $t_{n}$ in $\left[k 2^{-j},(k+1) 2^{-j}\right]$ as follows:

$$
t_{0}=(k+1) 2^{-j}
$$

and

$$
t_{n}=\max \left\{t \leq t_{n-1}:\left|\lambda(t)-\lambda\left(t_{n-1}\right)\right|=2^{-j / 2}\right\} .
$$

We continue until $t_{N} \leq k 2^{-j}$, and then we redefine $t_{N}=k 2^{-j}$. We also define a subset of the complex plane (in contrast with the set $R_{j, k}$, which is a subset of the $x$-plane) by setting

$$
S=\left[-2^{-j / 2+2}, 2^{-j / 2+2}\right] \times\left[2^{-j / 2}, 2^{-j / 2+2}\right] .
$$

We will show that a.s.

$$
H\left(R_{j, k}\right) \subset \bigcup_{n=1}^{N}\left(\hat{f}_{t_{n}}(S)\right)^{2} .
$$

Here and in what follows we use the notation $A^{2}$ to mean $\left\{a^{2}: a \in A\right\}$ for sets $A \subset \mathbb{C}$. 
Let $(x, t) \in R_{j, k}$, that is, $2^{-j} \leq x \leq 2^{-j+1}$ and $t_{n+1} \leq t \leq t_{n}$ for some $n \leq N-1$. Recall that $H(x, t)=\left(\hat{f}_{t}(i \sqrt{x})\right)^{2}$, where $\hat{f}_{t}(z)=g_{t}^{-1}(z+\lambda(t))$. Now,

$$
\hat{f}_{t}(i \sqrt{x}) \in \hat{f}_{t_{n+1}}(S)
$$

if and only if

$$
\hat{f}_{t_{n+1}}^{-1}\left(\hat{f}_{t}(i \sqrt{x})\right) \in S
$$

which is equivalent to

$$
g_{t_{n+1}}\left(\hat{f}_{t}(i \sqrt{x})\right)-\lambda\left(t_{n+1}\right) \in S \text {. }
$$

We will show this last statement. Set $\phi(s)=g_{s}\left(\hat{f}_{t}(i \sqrt{x})\right)$ for $t_{n+1} \leq s \leq t$, and note that $\phi(t)=i \sqrt{x}+\lambda(t)$. From the Loewner equation we have that

$$
\partial_{s} \phi(s)=\frac{2}{\phi(s)-\lambda(s)} \text {. }
$$

Hence the imaginary part of $\phi$ is decreasing, yielding

$$
\operatorname{Im} \phi(s) \geq \operatorname{Im} \phi(t) \geq 2^{-j / 2} \text {. }
$$

This in turn implies that

$$
\left|\partial_{s} \phi(s)\right| \leq \frac{2}{\operatorname{Im}(\phi(s)-\lambda(s))} \leq 2^{j / 2+1},
$$

which further gives

$$
|\phi(s)-\phi(t)| \leq|s-t| 2^{j / 2+1} \leq 2^{-j / 2+1} .
$$

Now we are ready to examine the real and imaginary parts of $g_{t_{n+1}}\left(\hat{f}_{t}(i \sqrt{x})\right)-$ $\lambda\left(t_{n+1}\right)$. We begin with the imaginary part:

$$
\begin{aligned}
\operatorname{Im}\left(g_{t_{n+1}}\left(\hat{f}_{t}(i \sqrt{x})\right)-\lambda\left(t_{n+1}\right)\right) & =\operatorname{Im} \phi\left(t_{n+1}\right) \\
& \leq\left|\phi\left(t_{n+1}\right)-\phi(t)\right|+\operatorname{Im} \phi(t) \\
& \leq 2^{-j / 2+1}+2^{-j / 2+1 / 2} \\
& \leq 2^{-j / 2+2} .
\end{aligned}
$$

In addition, we saw that $\operatorname{Im} \phi\left(t_{n+1}\right) \geq 2^{-j / 2}$, which gives

$$
\operatorname{Im}\left(g_{t_{n+1}}\left(\hat{f}_{t}(i \sqrt{x})\right)-\lambda\left(t_{n+1}\right)\right) \in\left[2^{-j / 2}, 2^{-j / 2+2}\right] .
$$

Before we look at the real part, we should recall 2 facts. First by the definition of the times $t_{n}$, we have that $\left|\lambda(t)-\lambda\left(t_{n+1}\right)\right| \leq 2^{-j / 2+1}$. Also recall that $\operatorname{Re} \phi(t)=\lambda(t)$. Now,

$$
\begin{aligned}
\left|\operatorname{Re}\left(g_{t_{n+1}}\left(\hat{f}_{t}(i \sqrt{x})\right)-\lambda\left(t_{n+1}\right)\right)\right|= & \left|\operatorname{Re} \phi\left(t_{n+1}\right)-\lambda\left(t_{n+1}\right)\right| \\
\leq & \left|\phi\left(t_{n+1}\right)-\phi(t)\right| \\
& \quad+\left|\operatorname{Re}\left(\phi(t)-\lambda\left(t_{n+1}\right)\right)\right| \\
\leq & 2^{-j / 2+1}+2^{-j / 2+1} \\
\leq & 2^{-j / 2+2} .
\end{aligned}
$$

Putting this together with the imaginary piece, we have just proved that

$$
g_{t_{n+1}}\left(\hat{f}_{t}(i \sqrt{x})\right)-\lambda\left(t_{n+1}\right) \in S
$$

which verifies equation (11). 


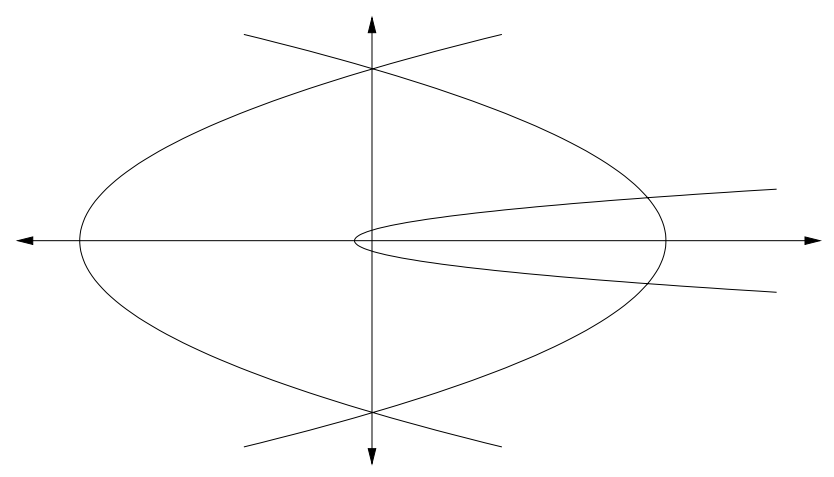

FiguRE 8 . The region $S^{2}$ is bounded by the three parabolas shown.

Our next goal is to use equation (11) to obtain (10). We begin by rewriting (11) as

$$
H\left(R_{j, k}\right) \subset \bigcup_{n=1}^{N}\left(\hat{h}_{t_{n}}\left(S^{2}\right)\right),
$$

where we have simply introduced the notation

$$
\hat{h}_{t}(z):=\left(\hat{f}_{t}(\sqrt{z})\right)^{2}=\left(g_{t}^{-1}(\sqrt{z}+\lambda(t))\right)^{2} .
$$

Since we will use this fact later, we note that for fixed $t, \hat{h}_{t}(z)=h_{t}(z)$ in distribution. The region $S^{2}$, pictured in Figure 8 , is bounded by the three parabolas $x=-2^{j-6} y^{2}+2^{-j+4}, x=2^{j-6} y^{2}-2^{-j+4}$, and $x=2^{j-2} y^{2}-2^{-j}$. It is simple to check that $\operatorname{diam}\left(S^{2}\right) \leq C 2^{-j}$. So then,

$$
\begin{aligned}
P\left[\operatorname{diam}\left(H\left(R_{j, k}\right)\right)>2^{-j \sigma}\right] \leq P & {\left[\sum_{n=1}^{N} \operatorname{diam}\left(\hat{h}_{t_{n}}\left(S^{2}\right)\right)>2^{-j \sigma}\right] } \\
\leq & P\left[C \sum_{n=1}^{N} \operatorname{diam}\left(S^{2}\right)\left|\hat{h}_{t_{n}}^{\prime}\left(-2^{-j}\right)\right|>2^{-j \sigma}\right] \\
\leq P & {\left[C N 2^{-j} \max _{1 \leq n \leq N}\left|\hat{h}_{t_{n}}^{\prime}\left(-2^{-j}\right)\right|>2^{-j \sigma}\right] } \\
\leq & P\left[N>j^{2}\right] \\
& +P\left[\max _{1 \leq n \leq N}\left|\hat{h}_{t_{n}}^{\prime}\left(-2^{-j}\right)\right|>C j^{-2} 2^{j(1-\sigma)}\right] .
\end{aligned}
$$

First, we will show that

$$
\sum_{j, k} P\left[N>j^{2}\right]<\infty
$$

and then we will complete the proof by showing that

$$
\sum_{j, k} P\left[\max _{1 \leq n \leq N}\left|\hat{h}_{t_{n}}^{\prime}\left(-2^{-j}\right)\right|>C j^{-2} 2^{j(1-\sigma)}\right]<\infty
$$

for $\sigma<\sigma(\kappa)$.

To show (12), note that $N$ roughly measures the number of oscillations of $\lambda(t)=$ $\sqrt{\kappa} B_{t}$ of size $2^{-j / 2}$ as $t$ decreases from $(k+1) 2^{-j}$ to $k 2^{-j}$. By the scale invariance 
of Brownian motion, this is the same as considering the number of oscillations of $\sqrt{\kappa} B_{t}$ of size 1 on the time interval $[0,1]$. Let

$$
p=P\left[\sqrt{\kappa}\left|B_{t}-B_{0}\right| \geq 1 \text { for some } t \in[0,1)\right]=P[N>1] .
$$

Now $0<p<1$, and further we see that

$$
P[N>n \mid N>n-1] \leq p,
$$

which implies that

$$
P[N>n] \leq p^{n}
$$

Therefore,

$$
\sum_{j, k} P\left[N>j^{2}\right] \leq \sum_{j, k} p^{j^{2}}=\sum_{j} 2^{j} p^{j^{2}}<\infty,
$$

giving equation (12).

Now we wish to verify (13). Set $\delta=C j^{-2} 2^{j(1-\sigma)}$. Then

$$
\begin{aligned}
P\left[\max _{1 \leq n \leq N}\left|\hat{h}_{t_{n}}^{\prime}\left(-2^{-j}\right)\right|>\delta\right] & =P\left[\exists n \in[1, N] \text { so that }\left|\hat{h}_{t_{n}}^{\prime}\left(-2^{-j}\right)\right|>\delta\right] \\
& \leq P\left[\bigcup_{n \geq 1}\left\{\left|\hat{h}_{t_{n}}^{\prime}\left(-2^{-j}\right)\right|>\delta \text { and } N \geq n\right\}\right] \\
& \leq \sum_{n \geq 1} P\left[\left|\hat{h}_{t_{n}}^{\prime}\left(-2^{-j}\right)\right|>\delta \text { and } N \geq n\right] \\
& =\sum_{n \geq 1} P\left[\left|\hat{h}_{t_{n}}^{\prime}\left(-2^{-j}\right)\right|>\delta \mid N \geq n\right] P[N \geq n] .
\end{aligned}
$$

Looking at the first term, we have

$$
\begin{aligned}
& P\left[\left|\hat{h}_{t_{n}}^{\prime}\left(-2^{-j}\right)\right|>\delta \mid N \geq n\right] \\
& \quad=\int_{k 2^{-j}}^{(k+1) 2^{-j}} P\left[\left|\hat{h}_{s}^{\prime}\left(-2^{-j}\right)\right|>\delta \mid t_{n}=s \text { and } N \geq n\right] P\left[t_{n} \in d s\right] \\
& \quad=\int_{k 2^{-j}}^{(k+1) 2^{-j}} P\left[\left|\hat{h}_{s}^{\prime}\left(-2^{-j}\right)\right|>\delta\right] P\left[t_{n} \in d s\right] \\
& \quad \leq \max _{0 \leq s \leq 1} P\left[\left|\hat{h}_{s}^{\prime}\left(-2^{-j}\right)\right|>\delta\right] .
\end{aligned}
$$

The second equality results from the independence of the events $\left\{\left|\hat{h}_{s}^{\prime}\left(-2^{-j}\right)\right|>\delta\right\}$ and $\left\{t_{n}=s\right.$ and $\left.N \geq n\right\}$. We have this independence because of the independent increments of Brownian motion and the fact that the first event is determined by Brownian motion on the time interval $[0, s]$, while the second event depends only on Brownian motion on the time interval $\left[s, t_{0}\right]$. Notice that $\sum_{n \geq 1} P[N \geq n] \leq$ $\sum_{n \geq 1} p^{n-1}<\infty$. Therefore,

$$
\begin{aligned}
P\left[\max _{1 \leq n \leq N}\left|\hat{h}_{t_{n}}^{\prime}\left(-2^{-j}\right)\right|>\delta\right] & \leq \sum_{n \geq 1} \max _{0 \leq s \leq 1} P\left[\left|\hat{h}_{s}^{\prime}\left(-2^{-j}\right)\right|>\delta\right] P[N \geq n] \\
& \leq C \max _{0 \leq s \leq 1} P\left[\left|\hat{h}_{s}^{\prime}\left(-2^{-j}\right)\right|>\delta\right] \\
& =C \max _{0 \leq s \leq 1} P\left[\left|h_{s}^{\prime}\left(-2^{-j}\right)\right|>\delta\right] .
\end{aligned}
$$


We obtain the final equality from the fact that $\hat{h}_{s}(z)$ is equal to $h_{s}(z)$ in distribution for fixed $s$.

We are now close to our goal of verifying equation (13). It remains to apply Lemmas 2 and 3 and then to analyze what values of $\sigma$ give rise to a convergent sum. From Lemma 2 we obtain that

$$
\sum_{j, k} P\left[\max _{1 \leq n \leq N}\left|\hat{h}_{t_{n}}^{\prime}\left(-2^{-j}\right)\right|>\delta\right] \leq C \sum_{j, k}\left(M^{\alpha} \delta^{-\alpha} 2^{j L / 2}+M^{-\mu} \log \left(C 2^{j / 2}\right)\right)
$$

for $\alpha, \mu, M$ positive and $L, b$ nonnegative with $L=\frac{\kappa}{2} b+\frac{\kappa}{4} \alpha$ and $-b^{2}+$ $\left(\frac{4}{k}+1-\alpha\right) b+\frac{\alpha}{2}-\frac{\alpha^{2}}{4}=0$. Recall that $\delta=C j^{-2} 2^{j(1-\sigma)}$ and that for fixed $j$, there are $2^{j}$ values for $k$. Also, set $M=2^{m j}$. Then equation (14) becomes

$$
\begin{aligned}
\sum_{j, k} P\left[\max _{1 \leq n \leq N}\left|\hat{h}_{t_{n}}^{\prime}\left(-2^{-j}\right)\right|>\delta\right] \leq & C \sum_{j} j^{2 \alpha} 2^{j(1+2 \alpha m-\alpha(1-\sigma)+L / 2)} \\
& +C \sum_{j} j 2^{j(1-\mu m)}
\end{aligned}
$$

We wish to show that the right hand side of this is finite for $\sigma<\sigma(\kappa)$. If

$$
1-\alpha(1-\sigma)+\frac{L}{2}<0,
$$

then by taking $m$ small enough the first sum will be finite. Once $m$ is fixed, we take $\mu>\frac{1}{m}$, which ensures that the second sum will be finite as well. Solving equation (15) for $\sigma$ gives

$$
\sigma<1-\frac{1+\frac{L}{2}}{\alpha}=1-\frac{1}{8 \alpha}\left(12+\kappa \pm \sqrt{(4+\kappa)^{2}-8 \kappa \alpha}\right) .
$$

For $\alpha>0$, the right hand side of (16) is maximized when the minus sign is chosen and when

$$
\alpha=\frac{1}{\kappa}(-4(\kappa+8)+(\kappa+12) \sqrt{\kappa+8}) .
$$

With this value of $\alpha$, equation (16) becomes

$$
\sigma<1-\frac{\kappa}{2 \kappa+24-8 \sqrt{\kappa+8}}
$$

as desired. Note that this is only valid as long as the value of $b$ corresponding to (17) is non-negative, and this occurs for $\kappa \leq-2+2 \sqrt{5}$. For $\kappa>-2+2 \sqrt{5}$, we must apply Lemma 3 instead of Lemma 2 using $D=j$. In this case we argue as above to finish the proof.

\section{REFERENCES}

1. V. Beffara, The dimension of the SLE curves, arXiv:math.PR/0211322.

2. D. Beliaev, Harmonic measure on random fractals, Royal Institute of Technology, Stockholm, 2005.

3. I. Binder and B. Duplantier, Harmonic measure and winding of conformally invariant curves, Physical Review Letters 89 (2002), no. 26.

4. I. Gruzberg and L. Kadanoff, The Loewner equation: maps and shapes, J. Statist. Phys. 114 (2004), no. 5-6, 1183-1198. MR2039475 (2005f:81210)

5. G. Lawler, O. Schramm, and W. Werner, Values of Brownian intersection exponents. I. Halfplane exponents, Acta Math. 187 (2001), no. 2, 237-273. MR1879850 (2002m:60159a)

6. Conformal invariance of planar loop-erased random walks and uniform spanning trees, Ann. Probab. 32 (2004), no. 1B, 939-995. MR2044671(2005f:82043) 
7. J. Lind, A sharp condition for the Loewner equation to generate slits, Ann. Acad. Sci. Fenn. Math. 30 (2005), no. 1, 143-158. MR2140303 (2006b:30013)

8. B. Oksendal, Stochastic differential equations, Springer, 1995. MR 1411679 (97f:60114)

9. S. Rohde and O. Schramm, Basic properties of SLE, Ann. of Math. (2) 161 (2005), no. 2, 883-924. MR2153402 (2006f:60093)

10. O. Schramm, Scaling limits of loop-erased random walks and uniform spanning trees, Israel J. Math. 118 (2000), 221-288. MR.1776084 (2001m:60227)

Department of Mathematics, Belmont University, 1900 Belmont Boulevard, NashVILle, Tennessee 37212 